\title{
Geodésicas ecuatoriales en espacio-tiempos conformestáticos axialmente simétricos producidos por discos relativistas de Kuzmin-Toomre con Halo
}

\section{Equatorial Geodesics in Axially Symmetric Confor- mastatic Spacetimes Produced by Relativistic Kuz- min-Toomre Disks with a Halo}

Recibido: 20 de noviembre de 2020. Recibido en revisión: 04 de diciembre de 2020. Aceptado:08 de febrero de 2021. DOl: https://doi.org/10.33132/27114260.1910 Luis Carlos Jaimes Supelano ${ }^{11}$

\section{Resumen}

Einstein formula la teoría de la relatividad general y cambia la forma de entender el universo heredada por Newton cientos de años atrás, esta nueva interpretación del aparato estelar invita a obtener soluciones al engranaje matemático de la relatividad general. Una de estas soluciones exactas provoca el presente trabajo, una solución estática — específicamente, la solución de Weyl - cuya solución general se puede escribir en coordenadas cilíndricas que conduce al subconjunto de soluciones asintóticamente planas denominadas métrica conformestática. Dada esta configuración de espacio-tiempo fue posible el estudio del movimiento de partículas en campos gravitacionales generados por fuentes axialmente simétricas, como los mostrados por la familia de discos delgados relativistas de Kuzmin-Toomre inmersos en halos, cuyos modelos representan soluciones a las ecuaciones de Einstein para un espacio-tiempo conformestático y con simetría axial. Posteriormente, se realizó un análisis gráfico del potencial efectivo para el plano ecuatorial, se resolvieron las

11 Escuela de Física. Universidad Industrial de Santander. Bucaramanga, Colombia. Luisjaimes1808@gamail.com 
ecuaciones de las geodésicas en dicho plano y se determinaron las posibles trayectorias de las partículas masivas.

\section{Abstract}

Einstein formulates the theory of general relativity and changes the way of understanding the universe inherited from Newton hundreds of years ago; this new interpretation of the star system entices us to solve the mathematical gear of general relativity. One of these exact solutions gives rise to the present work: the Weyl metrics, whose general solution can be written in cylindrical coordinates leading to the subset of asymptotically planar solutions called the conformastatic metrics. Given this spacetime configuration, it was possible to study the motion of particles in gravitational fields generated by axially symmetric sources, such as those shown by the Kuzmin-Toomre family of relativistic thin discs surrounded by a halo. Those models represent solutions to Einstein's equations for a conformastatic spacetime with axial symmetry. Subsequently, we made a graphical analysis of the effective potential for the equatorial plane, worked out the equations of the geodesics in this plane, and determined the potential trajectories of massive particles.

\section{Introducción}

Antes de 1915, la mecánica celeste se remonta al físico y matemático Isaac Newton que define la gravedad como una fuerza con la que las masas actúan unas sobre otras, derivando en la aceleración de estos cuerpos. Esto era cierto incluso en la teoría especial de la relatividad, los cuerpos moviéndose por fuerzas atractivas y repulsivas sin que el tiempo y el espacio se vieran afectados. Era lógico especular que el espacio y el tiempo continuaban sin ninguna alteración, sin embargo, es bastante diferente en la teo- ría de la relatividad general (Prasanna, 2016), basada en la revolucionaria interpretación que la gravedad no es una fuerza, sino una consecuencia de la curvatura del espacio-tiempo por la distribución de masa y energía en él, razón por la cual, los cuerpos no siguen órbitas curvas por una fuerza llamada gravedad, se mueven en órbitas curvas porque siguen lo más cercano a un camino recto en un espacio deformado, camino que se denomina geodésica (Ohanian \& Nordtvedt, 1977). Las partículas masivas y 
los rayos de luz también deben seguir las geodésicas en el espacio curvado, por esta razón, analizar trayectorias en una configuración específica del espacio-tiempo es de interés físico, ya que la forma en que orbiten estas partículas permite deducir propiedades del campo gravitatorio.

En busca de modelar los diferentes fenómenos de la naturaleza, en particular configuraciones discoidales de materia como las presentes en nebulosas y galaxias (Jones \& Lambourne, 2004) y de analizar las trayectorias que siguen las partículas en estas, es posible comenzar el estudio con el espacio-tiempo plano y buscar ecuaciones de campo que describan el campo gravitacional aproximándose al espacio de Minkowski. Una forma de realizar este análisis es mediante una configuración del espacio que permita ignorar la materia distante al punto de evaluación y omitir la curvatura presente como la métrica conformestática (Stephani et al., 2003), derivada de una solución estática a las ecuaciones de Einstein (Valiente Kroon, 2016) y conformalmente plana en su parte espacial. En relatividad general, soluciones estacionarias axialmente simétricas de las ecuaciones de Einstein juegan un papel crucial para la descripción del campo gravitacional de objetos astrofísicos cuya fuente de materia no rota (Synge, 1958).

Una de esas soluciones estáticas con simetría axial, corresponde a la configuración del espacio-tiempo conformestático cuya importancia radica en que la geometría del espacio es asintóticamente plana y puede ser usada para múltiples propósitos astrofísicos como el análisis de fuentes discoidales de polvo en movimiento constante (Katz et al., 1999), discos finitos cargados (González et al., 2008), sistema disco-halo en presencia de un campo electromagnético (Gutiérrez-Piñeres et al., 2013), una expresión no estándar del perihelio de pequeñas partículas en el sistema solar (Capistrano et al., 2014) y la superposición analítica de un agujero negro cargado con un disco anular de polvo extremo (Navarro et al., 2016).

Caracterizado el campo gravitacional, la investigación se dirige a estudiar las trayectorias que siguen las partículas masivas en estos campos. Varios autores dedicaron su conocimiento a calcular y analizar geodésicas en diferentes configuraciones del espacio-tiempo como lo hacen en la métrica de Schwarzschild (Hackmann \& Lämmerzahl, 2008) que permite modelar agujeros negros o en la métrica de Kerr que permite caracte- 
rizar agujeros negros rotantes (Wilkins, 1972). Del mismo modo, otras configuraciones menos populares presentan resultados interesantes de geodésicas como los que se evidencian en el espacio de Kasner (Harvey, 1989), Godel (Camci, 2014) o Bardeen (Zhou et al., 2012).

En la sección I se introducen los conceptos básicos de la configuración conformestática del espacio-tiempo derivado de las soluciones estáticas con simetría axial; se define matemáticamente el movimiento de partículas de prueba masivas en el plano ecuatorial mediante el formalismo Lagrangiano, se obtienen las expresiones para el potencial efectivo y las ecuaciones de movimiento de órbitas no circulares, basados en la formulación adimensional de todas las expresiones matemáticas. En la sección II se presenta un modelo relativista que describe discos delgados inmersos en un halo estelar, derivado de una familia de soluciones en la cual el potencial gravitacional está completamente determinado, es asintóticamente plano, libre de singularidades, y satisface todas las condiciones de energía. En la sección III se realiza el análisis gráfico del potencial efectivo de los primeros tres términos $(\mathrm{n}=0,1,2)$ de la familia de soluciones de Kuzmin-Toomre variando el momento angular de la partícula de prueba, se re- suelve numéricamente la ecuación de movimiento para los diferentes valores de los parámetros involucrados; se grafican las geodésicas de estas partículas, interpreta físicamente y se realiza un análisis cualitativo de estos resultados. Finalmente destaca una serie de conclusiones que se deducen del trabajo realizado.

\section{Movimiento de una partícula de prueba}

El elemento de línea de la métrica conformestática (González et al., 2008) en coordenadas cilíndricas es:

$$
d s^{2}=-e^{2 \psi} d t^{2}+e^{2 \psi}\left[d \rho^{2}+\rho^{2} d \varphi^{2}+d z^{2}\right],
$$

donde la función $\psi$ depende solamente de $(\rho, z)$. Los rangos de dichas coordenadas son estándares y se utiliza la convención de signos desarrollado en el lenguaje cuadrivectorial de Minkowski.

Para la geometría conformestática el lagrangiano corresponde a:

$$
2 \mathcal{L}=-e^{2 \psi} t^{2}+e^{2 \psi}\left[\rho^{2}+\rho^{2} \varphi^{2}+z^{2}\right],
$$

donde el punto denota derivación con respecto al parámetro afín $\tau$. Se introducen las constantes de movimiento que corresponden a la energía $-E=\frac{\partial \mathcal{L}}{\partial t}$ y el momento angular por unidad de masa $\ell=\frac{\partial \mathcal{L}}{\partial \varphi}$, que permiten comprender el comportamiento de la fuente gravita- 
toria (Scheck, 2010). El interés se concentra en las trayectorias sobre el plano ecuatorial, por tanto, la ecuación 2 se reduce a:

$$
2 \mathcal{L}=\frac{E^{2}}{e^{2 \psi}}+e^{-2 \psi} \rho^{2}+\frac{\ell^{2}}{\rho^{2}},(3)
$$

donde se impone una condición para distinguir el tipo de partícula analizada

$$
2 \mathcal{L}=\epsilon^{2}=0,-1,{ }_{(4)}
$$

con $\epsilon=0$ para el análisis de geodésicas tipo luz y $\epsilon=-1$ para geodésicas tipo tiempo. La órbita circular está determinada por las condiciones $\rho=c t e$ y $\rho=0$ que reduce la ecuación 3 a:

$$
\frac{E^{2}}{2}=\frac{\rho^{2}}{2}+\frac{e^{2 \psi}}{2} e^{-2 \psi}\left[\epsilon^{2}+\frac{\ell^{2}}{\rho^{2}} e^{2 \psi}\right],,(5)
$$

donde se define la expresión denominada potencial efectivo (Taylor, 2005)

$$
V_{e f f}=e^{2 \psi}\left[\epsilon^{2}+\frac{\ell^{2}}{\rho^{2}} e^{2 \psi}\right]
$$

el cual es función $\rho$ de y permite reinterpretar la definición de energía transformando el problema en un problema unidimensional; así, la investigación del movimiento de partículas de prueba en el espacio-tiempo conformestático se reduce al estudio del movimiento en el potencial efectivo Veff. Para efectos de la presente investigación, se analizará el movimiento de las partículas tipo tiem- po, pero es posible extender el análisis a las partículas tipo luz o fotones (Jaimes, 2018).

\section{Órbitas no circulares para partículas masivas}

Una partícula de prueba cuyo efecto gravitacional es insignificante, puede moverse en el espacio-tiempo conformestático en una variedad de órbitas posibles que se derivan resolviendo las ecuaciones de movimiento apropiadas, lo que es equivalente a encontrar las geodésicas en el espacio-tiempo. Es conocido que la geometría del espacio-tiempo puede ser caracterizada por el movimiento de partículas de prueba que se mueven a lo largo de las geodésicas en la geometría dada, tanto partículas masivas que se mueven en geodésicas tipo tiempo como rayos de luz que se mueven en geodésicas nulas. Las órbitas en el plano ecuatorial están determinadas por las coordenadas radial $\rho$ y azimutal $\varphi$ que se obtienen mediante:

$$
\begin{gathered}
\rho=\mathrm{E}^{2}-\mathrm{e}^{2} \psi \epsilon^{2}-\ell^{2} / \rho^{2},(7) \\
\varphi=\ell \mathrm{e}^{2 \psi} \rho^{-2}
\end{gathered}
$$

que corresponden a un sistema de ecuaciones diferenciales de primer orden que determinan las geodésicas en el plano $\mathrm{z}=0$. 
Para el movimiento de partículas de prueba en el plano $\mathrm{z}=0$ se necesitan las coordenadas $\rho$ у $\varphi$. Matemáticamente, el sistema que describe el movimiento está formado por dos ecuaciones. La ecuación 7 muestra la constante de movimiento momento angular por unidad de masa y establece que todo el movimiento permanece en el plano ecuatorial. Por analogía con el problema clásico se interpretan los términos como la energía cinética radial, la energía rotacional y la energía potencial. La ecuación 8 es otra forma de escribir la ecuación 6 y se obtiene directamente del elemento de línea (ecuación 2), de esta forma, para un conjunto de dos ecuaciones y dos variables, el sistema está bien determinado y tiene solución numérica.

\section{Discos relativistas de Kuzmin-Toomre}

El análisis de la teoría del potencial newtoniano de discos infinitesimalmente delgados permite generar potenciales de discos axisimétricos, estos resultados se pueden utilizar para construir secuencias infinitas de nuevas soluciones de ecuaciones de Einstein que describen discos de masa finita. A grandes distancias, estos discos se vuelven newtonianos, pero en sus regiones centrales exhiben características relativistas. En particular, se construyeron espacios-tiempos mediante una familia de soluciones exactas denominadas discos relativistas de Kuzmin-Toomre cuyas propiedades se mencionan y se ilustran.

Una solución al problema de calcular el potencial gravitacional generado por una distribución en forma de disco delgado de materia fue propuesta por primera vez por Kuzmin y después generalizada por Toomre en lo que se conoce como el modelo de Kuzmin-Toomre (Binney \& Scott, 2011). El modelo está definido por una solución particular de la ecuación de Laplace que cumple las condiciones de energía

$$
\nabla^{2} \psi=\mathrm{k} \nabla \psi \cdot \nabla \psi,(9)
$$

con $\mathrm{k}>1$; Si k tiende al infinito, el fluido en el halo estará hecho de polvo, si $\mathrm{k}$ es igual a 1 , describirá un fluido hecho de radiación (Pimentel, 2012). Es de gran importancia comprender que se debe encontrar otra función para modelar el potencial gravitacional, por tanto, la ecuación 9 se puede reescribir como:

$$
\nabla^{2}\left(\mathrm{e}^{-\mathrm{k} \psi}\right)=0,(10)
$$

de modo que la función sea solución a la ecuación de Laplace ya que esta ecuación juega un papel importante en el desarrollo de los campos vectoriales que 
derivan de un potencial gravitatorio, además de describir procesos estacionarios.

Por características de la métrica conformestática, el espacio-tiempo tiende a Minkowski en el infinito, lo que implica que debe ser asintóticamente plano, lo que transforma la ecuación 10 en:

$$
\mathrm{e}-\mathrm{k} \psi=1-\Phi,(11)
$$

que permite relacionar los términos de la familia de soluciones de Kuzmin-Toomre con la función $\Phi$ deseada. La función posee simetría de reflexión alrededor del plano del disco, es decir:

$$
\Phi(\rho, z)=\Phi(\rho,-z),(12)
$$

que indica que el potencial gravitacional sobre la distribución de masa $\mathrm{z}>$ 0 , posee las mismas propiedades que en $\mathrm{z}<0$. El modelo tiene una expresión general definida por:

$$
\Phi_{n}(R, \theta)=-\sum_{\ell=0}^{n} \frac{C_{\ell}}{R^{\ell+1}} P_{\ell}(\cos \theta),,(13)
$$

donde $C_{\ell}$ son constantes, $P_{\ell}(\cos \theta)$ son los polinomios de Legendre (Arfken et al., 2013) y

$$
\begin{gathered}
R=\sqrt{\rho^{2}+z^{2}} \\
\cos (\theta)=\frac{z}{R}
\end{gathered}
$$

Ahora, como esta solución y todas sus derivadas son continuas, se introdu- ce una transformación sobre la coordenada $\mathrm{z}$

$$
\mathrm{z} \rightarrow|\mathrm{z}|+\mathrm{d},(16)
$$

donde $d$ es una constante positiva y determina el modelo de discos de Kuzmin-Toomre que presenta un número infinito de funciones que describe exitosamente un modelo de potencial gravitacional para distribuciones masivas aplanadas. Para esta investigación se trabajaron los primeros tres términos de la familia de soluciones, es decir, la solución para los casos $n=0,1,2$ (Jaimes, 2018).

\section{Análisis de resultados}

Es posible reducir el problema de una partícula al análisis de un potencial central (ecuación 6) cuya dinámica aporta mucha información sobre las trayectorias en dicho potencial, que es el objetivo de esta investigación. Para obtener los resultados que se van a exponer, se resolvieron las ecuaciones de movimiento mediante un software de cálculo simbólico como MAPLE y se obtuvo una serie de resultados numéricos que fueron posteriormente graficados y analizados cualitativamente.

La forma de interpretar los datos y de estudiarlos consistió en primera instancia en graficar el potencial efectivo 


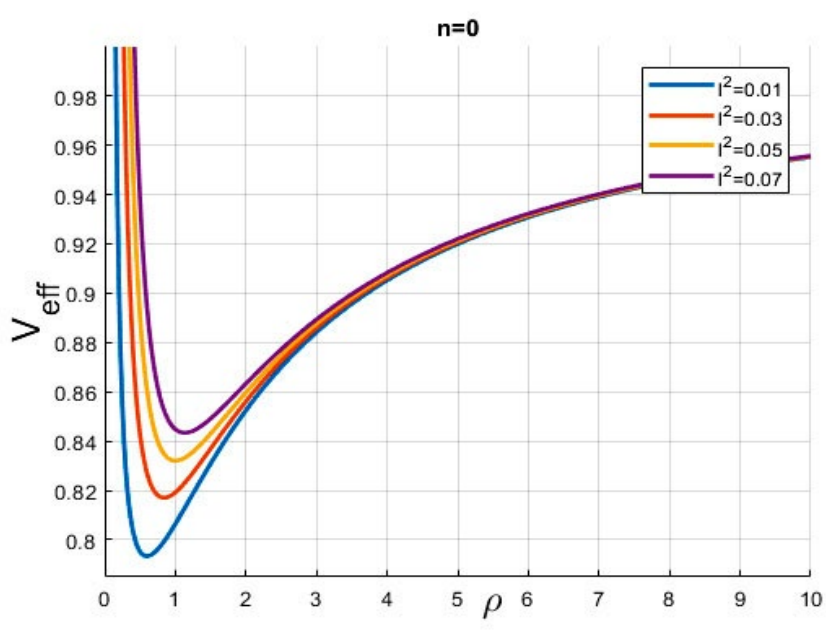

Figura 1. Cambios en el potencial efectivo para el modelo $\mathrm{n}=0$ de partículas masivas cuando varía el valor del momento angular $\ell^{2} \operatorname{con} \mathrm{A}_{0}=2 \mathrm{y} \mathrm{k}=7.9$. Fuente: elaboración propia.

de la partícula y observar su variación en función del momento angular, luego, se identifican zonas energéticas principales y se analizan cuáles son las posibles trayectorias en estas zonas, realizando las respectivas gráficas de las geodésicas de la partícula y finalmente se hace una interpretación física del fenómeno.

En la figura 1 se grafica el potencial efectivo para el término $\mathrm{n}=0 \mathrm{y}$ se varía el momento angular de las partículas de prueba, se reconocen algunos puntos importantes como el pozo de potencial, la distancia entre el potencial efectivo y el eje $\rho$ y el mínimo del potencial efectivo. En primera instancia una partícula masiva que tenga una energía superior al pozo viene de un punto lejano, se acerca al centro del potencial hasta una distancia determinada por el efecto de la fuerza centrífuga debido al momento angular, rebota en una especie de barrera de potencial y como no tiene cota superior se dirige al infinito.

Si la partícula tiene una energía que la confina dentro del pozo de potencial, tendrá una cota inferior y superior, lo que indica que va a fluctuar su trayectoria. Dependiendo de su momento angular, tendrá cotas inferiores más lejanas del centro de potencial y en general una distancia máxima a la que llega la 
partícula que varía en función de las condiciones de energía iniciales. Si la partícula tiene una energía que la ubica exactamente en el mínimo del potencial, esta tendrá una trayectoria circular estable. Ahora se grafican las geodésicas de la partícula masiva con momentos angulares $\ell^{2}=0.05$ y $\ell^{2}=0.1$ para el modelo $\mathrm{n}=0$.

En la figura 2 y figura 3, la órbita circular estable es representada por un círculo rojo y las trayectorias por trazos azules. Se observan situaciones características de las órbitas que varían según el momento angular; el radio de la órbita circular es más grande a medida que el momento angular crece, la cota inferior y superior crecen, es decir, cada vez están más alejadas del centro del potencial, y el círculo central también crece. Ahora se analiza el comportamiento de los modelos $n=1$ y $n=2$.

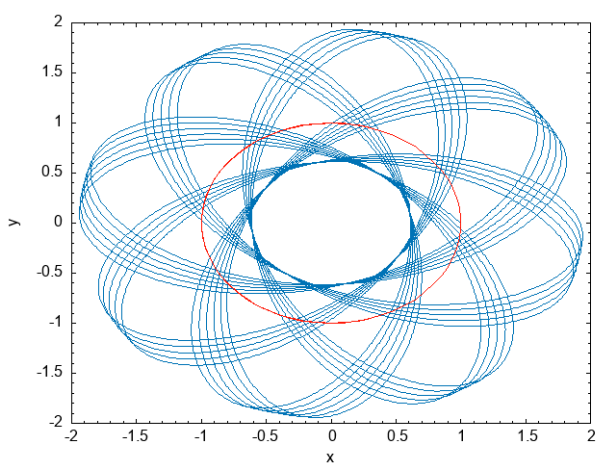

Figura 2. Trayectorias acotadas de partículas masivas con $\ell^{2}=0.05$ para el modelo de $\mathrm{n}=0$. Fuente: elaboración propia.

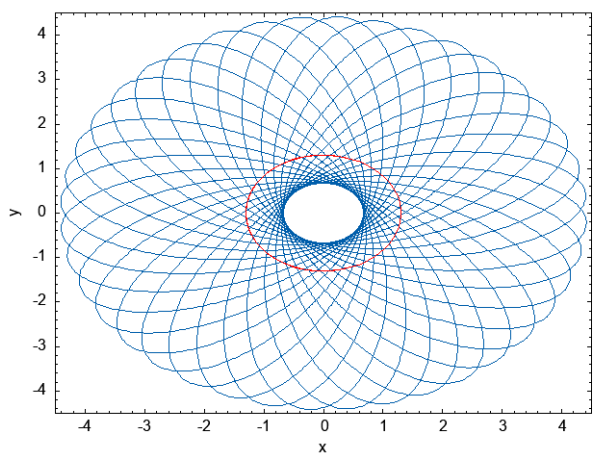

Figura 3. Trayectorias acotadas de partículas masivas $\operatorname{con} \ell^{2}=0.1$ para el modelo de $\mathrm{n}=0$. Fuente: elaboración propia. 


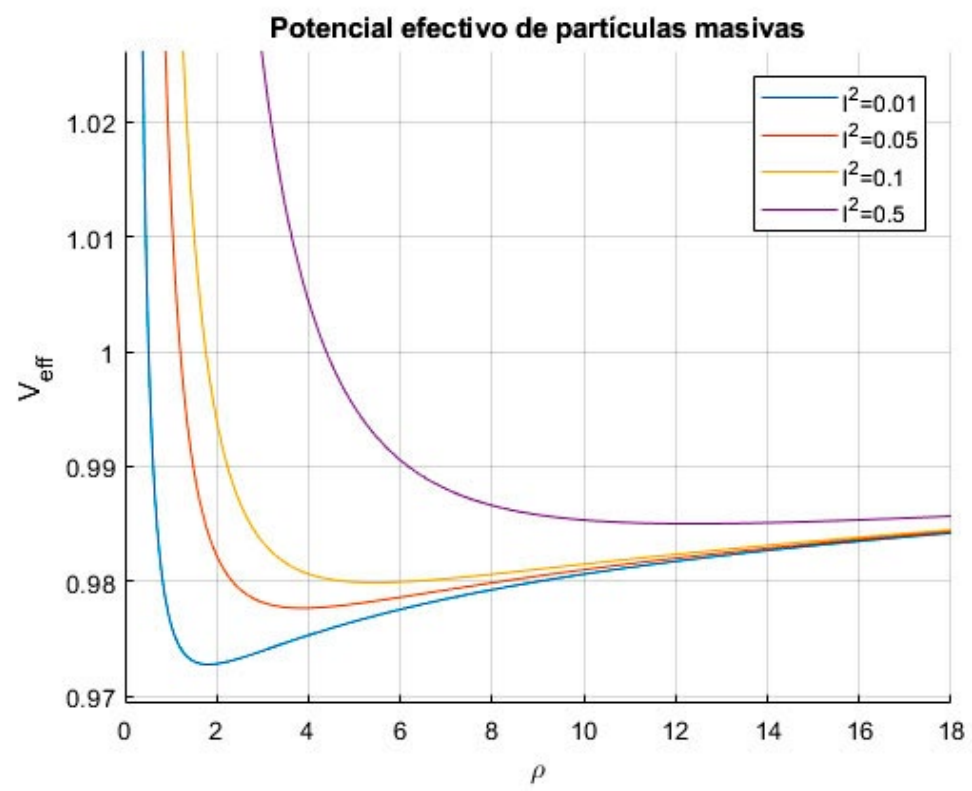

Figura 4. Cambios en el potencial efectivo para el modelo $\mathrm{n}=1$ de partículas masivas cuando varía el valor del momento angular $\ell^{2} \operatorname{con} A_{0}=167, A_{1}=46, y k=292$.

Fuente: elaboración propia.

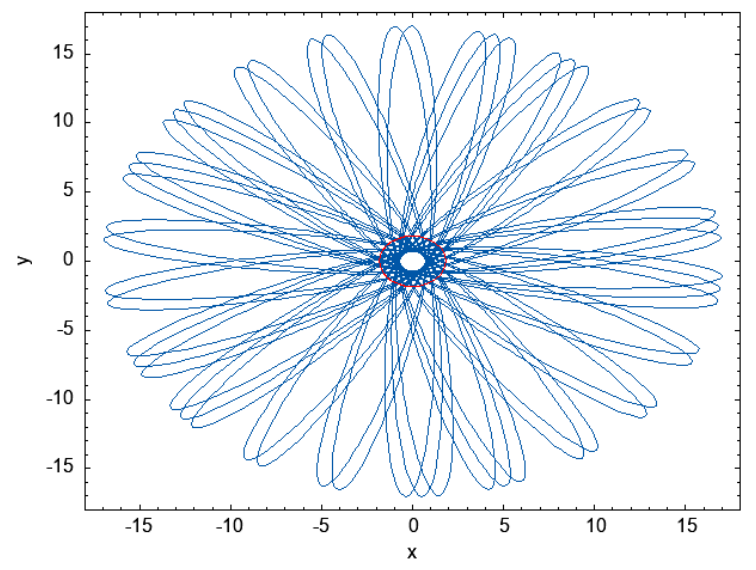

Figura 5. Trayectorias acotadas de partículas masivas $\operatorname{con} \ell^{2}=0.01$ para el modelo de $\mathrm{n}=1$. Fuente: elaboración propia. 


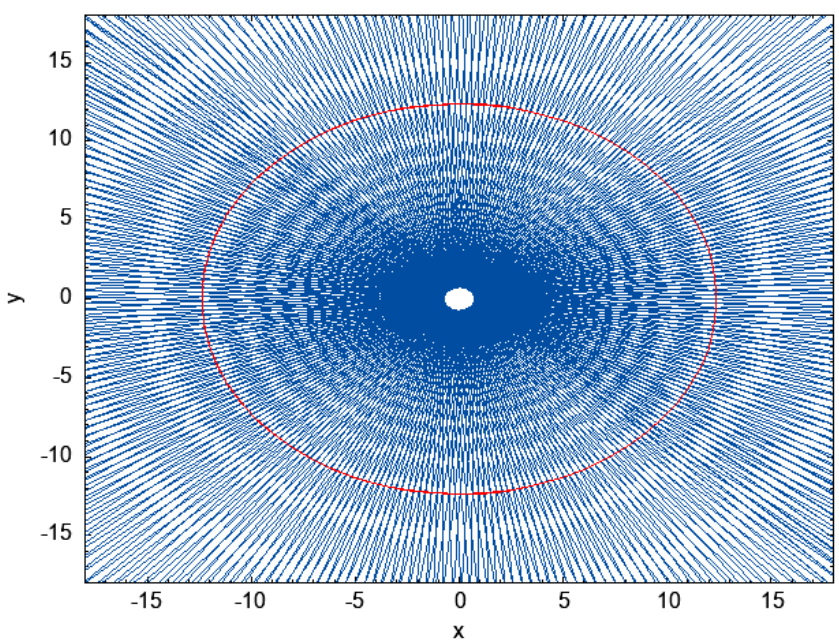

Figura 6. Trayectorias acotadas de partículas masivas $\operatorname{con} \ell^{2}=0.5$ para el modelo de $n=1$.

Fuente: elaboración propia.

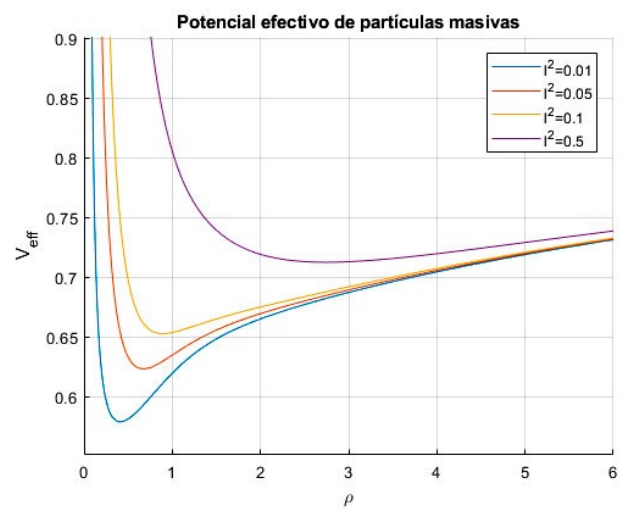

Figura 7. Cambios en el potencial efectivo para el modelo $n=2$ de partículas masivas cuando varía el valor del momento angular $\ell^{2} \operatorname{con} \mathrm{A}_{0}=100, \mathrm{~A}_{1}=-20, \mathrm{~A}_{2}=90 \mathrm{y} \mathrm{k}=18.12$.

Fuente: elaboración propia.

Para la familia de soluciones con $n=$ las órbitas son bastante pronunciadas. A 1 se puede observar que el potencial no tiene un pozo de potencial pronunciado, pero existen órbitas circulares estables, del potencial es mayor, así como el radio lo que indica que la cota mayor es bas- de las órbitas circulares estables. tante lejana del centro, razón por la cual, 


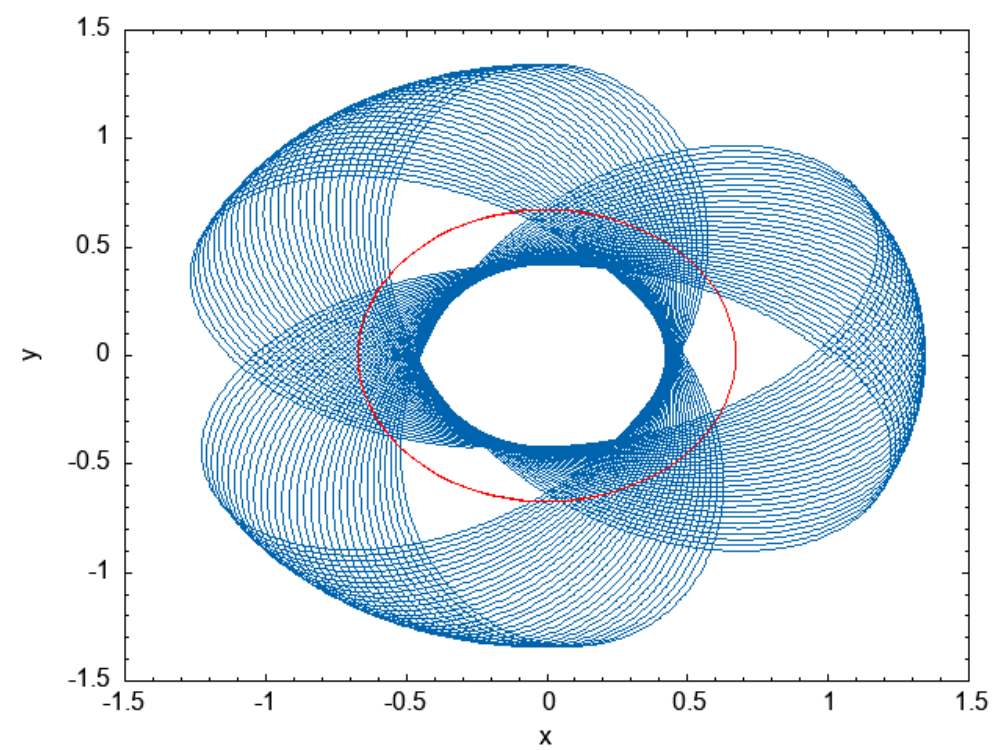

Figura 8. Trayectorias acotadas de partículas masivas $\operatorname{con} \ell^{2}=0.03$ para el modelo de $\mathrm{n}=2$. Fuente: elaboración propia.

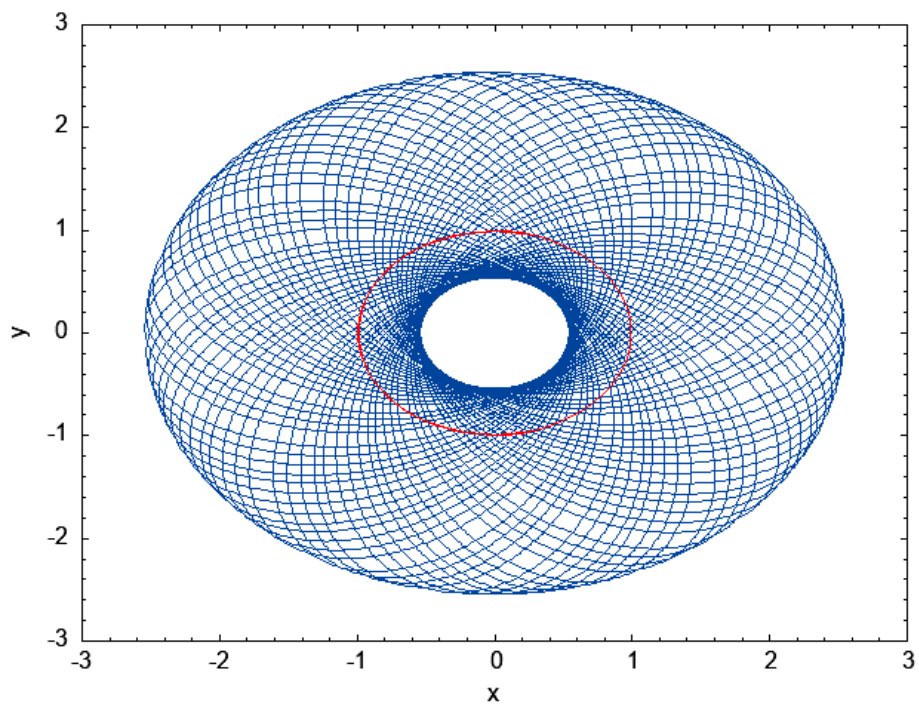

Figura 9. Trayectorias acotadas de partículas masivas $\operatorname{con} \ell^{2}=0.05$ para el modelo de $\mathrm{n}=2$. Fuente: elaboración propia. 
La familia de soluciones $\mathrm{n}=2$ tiene un comportamiento similar a $\mathrm{n}=0$ como lo señalan la figura 8 y figura 9 , ya que presenta un pozo de potencial un poco más profundo cuando el momento angular decrece, esto indica trayectorias definidas que tienen cota superior, un círculo central que denota la distancia mínima con respecto al origen del potencial y aún existen órbitas circulares estables.

\section{Conclusiones}

En este trabajo se ha realizado el estudio de órbitas en campos gravitacionales axialmente simétricos generados por fuentes discoidales de materia bajo la formulación Lagrangiana. El modelo de potencial elegido cumple con la condición de configuración de materia axial y además presenta simetría respecto al plano $\mathrm{z}=0$. Los modelos elegidos fueron los primeros tres términos de la familia de soluciones de discos relativistas de Kuzmin-Toomre que fueron generados a partir de un potencial generalizado de infinitos términos. A cada modelo del potencial se analizó la dinámica de las órbitas en el plano ecuatorial, se determinó la expresión del potencial efectivo y bajo un determinado conjunto de condiciones iniciales y parámetros se encontró la solu- ción de las ecuaciones de las geodésicas mediante una solución determinada por métodos numéricos.

El modelo estudiado se solucionó a partir del método Runge-Kutta-Fehlberg de quinto orden que es un algoritmo de análisis numérico para la resolución numérica de ecuaciones diferenciales ordinarias, lo que permite graficar las geodésicas de las diferentes partículas de prueba. De lo cual se concluyó de manera general para partículas masivas que las geodésicas son órbitas abiertas acotadas y contiene al menos una órbita circular estable. Es interesante encontrar que las órbitas circulares en el espacio-tiempo conformestático siempre será estable, ya que indistintamente de la elección del momento angular, el potencial efectivo tendrá un mínimo.

\section{Referencias}

Arfken, G. B., Weber, H. J., \& Harris, F. E. (2013). Mathematical Methods for Physicists. In Mathematical Methods for Physicists. https:// doi.org/10.1016/C2009-0-3062.9-7

Binney, J., \& Scott, T. (2011). Galactic dynamics. Contemporary Physics, 30(1), 65-67. https:// doi.org/10.1080/00107518908222591

Camci, U. (2014). Symmetries of geodesic motion in Gödel-type spacetimes. Journal of Cosmology and Astroparticle Physics, 
2014(7). https://doi.org/10.1088/1475$7516 / 2014 / 07 / 002$

Capistrano, A. J. S., Roque, W. L., \& Valada, R. S. (2014). Weyl conformastatic perihelion advance. Monthly Notices of the Royal Astronomical Society, 444(2), 1639-1646. https:// doi.org/10.1093/mnras/stu1567

González, G. A., Gutiérrez-Piñeres, A. C., \& Ospina, P. A. (2008). Finite axisymmetric charged dust disks in conformastatic spacetimes. Physical Review D - Particles, Fields, Gravitation and Cosmology, 78(6), 1-8. https:// doi.org/10.1103/PhysRevD.78.064058

Gutiérrez-Piñeres, A. C., González, G. A., \& Quevedo, H. (2013). Conformastatic disk-haloes in Einstein-Maxwell gravity. Physical Review D - Particles, Fields, Gravitation and Cosmology, 87(4), 1-12. https:// doi.org/10.1103/PhysRevD.87.044010

Hackmann, E., \& Lämmerzahl, C. (2008). Geodesic equation in Schwarzschild-(anti-) de Sitter space-times: Analytical solutions and applications. Physical Review D - Particles, Fields, Gravitation and Cosmology, 78(2), 1-22. https://doi.org/10.1103/PhysRevD.78.024035

Harvey, A. (1989). Geodesics in Kasner universes. 19(301), 6740. http://indication. bsnmedical.com/fileadmin/professionals/ PDFs/Acute Wound Care EBM/Bali D 2010 Clinical experiences with a Skin Stapler EWMA.pdf

Jaimes, L. C. (2018). Estudio de Geodésicas ecuatoriales en espacio-tiempos conformestáticos axialmente simétricos producidos por discos relativistas de Kuzmin-Toomre con Halo. Universidad Industrial de Santander. Escuela de física.

Jones, M. H., \& Lambourne, R. (2004). An Introduction to Galaxies and Cosmology. CUP.

Katz, J., Bicák, J., \& Lynden-Bell, D. (1999). Disc sources for conformastationary metrics. IOPScience, 4023.

Navarro, A., Lora-Clavijo, F. D., \& González, G. A. (2016). Relativistic static thin disks of polarized matter. Journal of Physics: Conference Series, 755(1). https://doi. org/10.1088/1742-6596/755/1/011001

Ohanian, H. C., \& Nordtvedt, K. (1977). Gravitation and Spacetime. In American Journal of Physics. https://doi.org/10.1119/1.10631

Pimentel, O. (2012). Construcción de modelos relativistas de discos delgados axialmente simétricos inmersos en un halo. Universidad Industrial de Santander.

Prasanna, A. R. (2016). Gravitation. In Gravitation. https://doi.org/10.1201/9781315368702

, F. (2010). Mechanics: From Newton's Laws to Deterministic Chaos. Springer-Verlag Berlin.

Stephani, H., Kramer, D., MacCallum, M., Hoenselaers, C., \& Herlt, E. (2003). Exact Solutions of Einstein's Field Equations. In Exact Solutions of Einstein's Field Equations. https://doi.org/10.1017/cbo9780511535185

Synge, J. (1958). Relativity: The special theory. Interscience Publishers.

Taylor, J. R. (2005). Classical Mechanics. University Science Books. 
Valiente Kroon, J. A. (2016). Conformal methods in general relativity. In Conformal Methods in General Relativity. https://doi.org/10.1017/ CBO9781139523950

Wilkins, D. C. (1972). Bound geodesics in the Kerr metric. Physical Review D, 5(4), 814-822. https://doi.org/10.1103/PhysRevD.5.814
Zhou, S., Chen, J., \& Wang, Y. (2012). Geodesic structure of test particle in bardeen spacetime. International Journal of Modern Physics D, 21(9), 1-13. https://doi.org/10.1142/ S0218271812500770 